\begin{tabular}{c} 
International Journal of Engineering \& Technology, $7(4.5)(2018) 511-513$ \\
SPC \\
International Journal of Engineering \& Technology \\
Website $\frac{w w w . s c i e n c e p u b c o . c o m / i n d e x . p h p / I J E T}{\text { Research paper }}$ \\
\hline
\end{tabular}

\title{
A simple and efficient wavelet approach for evaluating surface integral over curved domain
}

\author{
K. T. Shivaram ${ }^{1}$, S. Kiran ${ }^{2}$ \\ ${ }^{1}$ Department of Mathematics, Dayananda Sagar College of Engineering, Bangalore, Karnataka, India \\ ${ }^{2}$ Department of Mathematics, Sapthagiri College of Engineering, Bangalore, Karnataka, India \\ *Corresponding author E-mail: shivaramktshiv@gmail.com
}

\begin{abstract}
This paper presents, a simple and efficient wavelet approach for computing the surface integrals over irregular or curved dom ain, the limit of the integrals are nonlinear function are transformed to standard two square by using finite element basis function, Haar wavelet based integration technique is applied to evaluation of surface integral over curved domain, the computational efficiency of the method is illustrated with several numerical examples.
\end{abstract}

Keywords: Numerical Integration; Hear Wavelet Method; Curved Domain

\section{Introduction}

Numerical integration appears in many fields of science and engineering, to finding surface area, volume, length, momentum, mass, stiffness matrix, mass matrix, body force vector etc., In recent years wavelet bases integration approach is morepopular in the field of numerical approximation of surface and volume integral. From the literature review we may realize that a lot of works have been done in this area in terms of quadrature rule of numerical integration, A number of polynomial based Gauss Legendre quadrature rule, product of polynomial and logarithmic based Generalized Gaussian quadrature methodover various region have been discussed, such as triangle region [1-2], quadrilateral region [3], parabolic region [4], square region [5], circle [6], etc., numerical integration of arbitrary function over convex and non-convex region are evaluated numerically by cubic spline element method [7], convex and non convex region is divided into 4-node, 8-node quadrilateral elements are approximated Numerically in [8-9], very limited work is attempted in curved domain, recently numerical integration of arbitrary function over parabolic edges by Generalized Gaussian Where and Using the orthogonal basis of the Haar wavelet function can be expressed by Haar series function of infinite terms as Where For double integraldivided into 4-node, 8-node quadrilateral elements are approximated numerically in $[8,9]$, very limited work is attempted in curved domain, recently numerical integration ofarbitrary function over parabolic edges by Generalized Gaussian quadrature rule are carryout by [10], In this paper, we use Haar wavelet method to approximate the surface integral over curved domain or irregular domain.

\section{Mathematical preliminaries}

\subsection{Hear Wavelet method}

The explicit form of the function is defined as We shall be using this formula to evaluate the surface integral by Haar wavelet method

\subsection{Surface integral over $X Y$ - plane}

In this section is devoted to the numerical integration of arbitrary function over curved domain, Curved or irregular region is expressed as Where and are nonlinear function and $a$ and $b$ are constants are plotted in figure. 1 


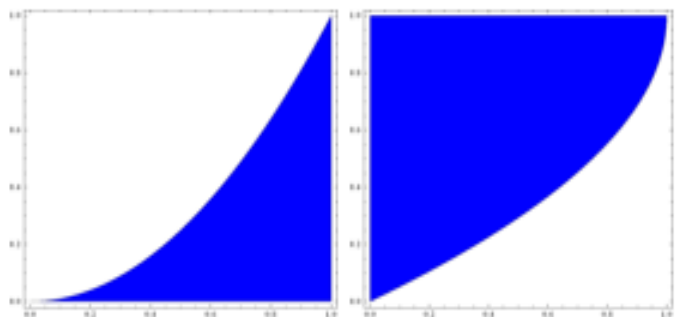

Tabl

\begin{tabular}{|c|c|c|c|}
\hline Exact value & Order N & Computed value & Error \\
\hline \multirow{4}{*}{$=0.0696617011$} & $\mathrm{~N}=10$ & 0.07088172435 & 0.00122 \\
\hline & $\mathrm{N}=100$ & 0.06967379875 & $1.2 \mathrm{E}-05$ \\
\hline & $\mathrm{N}=200$ & 0.06966468331 & $3 \mathrm{E}-06$ \\
\hline & $\mathrm{N}=10$ & 0.33506553638 & 0.000335 \\
\hline \multirow{3}{*}{$=0.3354005176$} & $\mathrm{~N}=100$ & 0.33539716912 & $3.35 \mathrm{E}-06$ \\
\hline & $\mathrm{N}=200$ & 0.33539967764 & $8.4 \mathrm{E}-07$ \\
\hline & $\mathrm{N}=10$ & 0.31357010110 & 0.00026 \\
\hline \multirow[t]{2}{*}{$=0.3133093585$} & $\begin{array}{l}\mathrm{N}=100 \\
\mathrm{~N}=200\end{array}$ & $\begin{array}{l}0.31331888372 \\
0.31331278756\end{array}$ & $\begin{array}{l}9.5 \mathrm{E}-06 \\
3.4 \mathrm{E}-06\end{array}$ \\
\hline & $\mathrm{N}=10$ & 0.73018819262 & 0.00097 \\
\hline$=0.7292185744$ & $\begin{array}{l}\mathrm{N}=100 \\
\mathrm{~N}=200 \\
\mathrm{~N}=10\end{array}$ & $\begin{array}{l}0.72924985935 \\
0.72922965894 \\
0.10206865780\end{array}$ & $\begin{array}{l}3.1 \mathrm{E}-05 \\
1.1 \mathrm{E}-05 \\
0.000802\end{array}$ \\
\hline$=0.1028703090$ & $\begin{array}{l}\mathrm{N}=100 \\
\mathrm{~N}=200 \\
\mathrm{~N}=10\end{array}$ & $\begin{array}{l}0.10286225153 \\
0.10286829387 \\
0.63509969592\end{array}$ & $\begin{array}{l}8.06 \mathrm{E}-06 \\
2.02 \mathrm{E}-06 \\
0.002337\end{array}$ \\
\hline \multirow{4}{*}{$=0.6374363872$} & $\mathrm{~N}=100$ & 0.63736328505 & $7.31 \mathrm{E}-05$ \\
\hline & $\mathrm{N}=200$ & 0.63741051888 & $2.59 \mathrm{E}-05$ \\
\hline & $\mathrm{N}=10$ & 0.30480315720 & 0.00044 \\
\hline & $\mathrm{N}=100$ & 0.30436590362 & $5.8 \mathrm{E}-06$ \\
\hline
\end{tabular}

The surface integral of eq.(5) can be transformed to square

\subsection{Consider the surface integral of arbitrary function}

Over the curved region is given by The surface integral of eq.(7) can be transformed to square

\section{Numerical integration over two dimensional curved region}

\subsection{Consider the surface integral of arbitrary function+}

Over the curved region The surface integral of eq. (3) can be transformed to square The Jacobian of the transformation is hence, Eq. (3) becomes

\subsection{Consider the surface integral of arbitrary function}

Over the curved region is given by the surface integral of eq. (5) can be transformed to square Hence, eq. (7) becomes.

\subsection{Consider the surface integral of arbitrary function}

Over the curved region is given by the surface integral of eq. (9) can be transformed to square and hence, eq. (9) becomes.

\subsection{Consider the surface integral of arbitrary function}

Over the curved region is given by The surface integral of eq. (11) can be transformed to square and, hence, eq. (11) becomes

\subsection{Consider the surface integral of arbitrary function}

Over the curved region and The surface integral of eq. (13) can be transformed to square We approximate the integral Eqs. (4), (6), (8), (10), (12), (14) by Applying eq. (2) of .Haar wavelet method is described in the.

\begin{tabular}{|c|c|c|c|}
\hline$=0.3043600979$ & $\mathrm{~N}=200$ & 0.30436170471 & $1.6 \mathrm{E}-06$ \\
\hline & $\mathrm{N}=10$ & 0.05372722301 & 0.00016 \\
\hline \multirow{3}{*}{$=0.05357142857$} & $\mathrm{~N}=100$ & 0.05357299091 & $1.6 \mathrm{E}-06$ \\
\hline & $\mathrm{N}=200$ & 0.05357181919 & 3.9E-07 \\
\hline & $\mathrm{N}=10$ & 0.93448217360 & 0.00016 \\
\hline \multirow{3}{*}{$=0.93432004929$} & $\mathrm{~N}=100$ & 0.93432167178 & $1.6 \mathrm{E}-06$ \\
\hline & $\mathrm{N}=200$ & 0.93432045875 & 4.1E-07 \\
\hline & $\mathrm{N}=10$ & 0.55263815130 & 0.000342 \\
\hline \multirow{4}{*}{$=0.5529797990$} & $\mathrm{~N}=100$ & 0.55297668185 & $3.12 \mathrm{E}-06$ \\
\hline & $\mathrm{N}=200$ & 0.55297902951 & $7.69 \mathrm{E}-07$ \\
\hline & $\mathrm{N}=10$ & 0.83312500000 & 0.000208 \\
\hline & $\mathrm{N}=100$ & 0.83333125000 & $2.08 \mathrm{E}-06$ \\
\hline \multirow{3}{*}{$=0.8333333333$} & $\mathrm{~N}=200$ & 0.83333281250 & $5.21 \mathrm{E}-07$ \\
\hline & $\mathrm{N}=10$ & 0.30216827585 & 0.000213 \\
\hline & $\mathrm{N}=100$ & 0.30237880968 & $2.14 \mathrm{E}-06$ \\
\hline$=0.3023809524$ & $\mathrm{~N}=200$ & 0.30238041666 & $5.36 \mathrm{E}-07$ \\
\hline
\end{tabular}

\section{Conclusions}

In this paper, Wavelet based integration method is applied for numerical integration of arbitrary function over curved or irregular domain, it converges to the exact value of the surface integral, sufficient value of order $\mathrm{N}$ for this proposed method. 


\section{References}

[1] D. A. Dunavant (1985), High degree efficient symmetrical Gaussian Quadrature rules for triangle, Int. J. Numer. Methods, Eng. 21, 11291148.

[2] G. Lague and R. Baldur (1977) Extended numerical integration method for triangular surfaces, Int. J. Numer. Methods Eng. 11, 388392.

[3] Md. S. Islam, M. Alamgir Hossain (2009), Numerical integrations over an arbitrary quadrilateral region, Appl. Math. Comput. 210, 515-524.

[4] K.T. Shivaram (2013), Gauss Legendre quadrature over a parabolic region, Int. J. Eng. Res. \& Tech, 10,927-931.

[5] K.T. Shivaram ( 2014), Numerical integration over an arbitrary rectangle and square region using Generalized Gaussian quadrature rules, International Journal of Mathematical Archive, 5, 1-5.

[6] K.T. Shivaram(2013), Gauss Legendre Quadrature over a unit circle International Journal of Engineering Research \& Technology, 2, 1043-1047.

[7] C.-J. Li, R.-H. Wang (2006), a new 8-node quadrilateral cubic spline finite element, J. Comput. Appl. Math.195, $54-65$. 